\title{
Swift XRT and UVOT deep observations of the high-energy peaked BL Lacertae object PKS 0548-322 close to its brightest state
}

\author{
M. Perri ${ }^{1}$, A. Maselli ${ }^{2}$, P. Giommi ${ }^{1,3}$, E. Massaro ${ }^{2}$, R. Nesci $^{2}$, A. Tramacere ${ }^{2}$, M. Capalbi ${ }^{1}$, G. Cusumano ${ }^{4}$, \\ G. Chincarini ${ }^{5,6}$, G. Tagliaferri ${ }^{5}$, D. N. Burrows ${ }^{7}$, D. V. Berk ${ }^{7}$, N. Gehrels ${ }^{8}$, and R. M. Sambruna ${ }^{8}$ \\ 1 ASI Science Data Center, via Galileo Galilei, 00044 Frascati, Italy \\ e-mail: perri@asdc.asi.it \\ 2 Dipartimento di Fisica, Università La Sapienza, Piazzale A. Moro 2, 00185 Roma, Italy \\ Agenzia Spaziale Italiana, Unità Osservazione dell’Universo, Viale Liegi 26, 00198 Roma, Italy \\ 4 INAF - Istituto di Astrofisica Spaziale e Fisica Cosmica, Sezione di Palermo, via La Malfa 153, 90146 Palermo, Italy \\ 5 INAF - Osservatorio Astronomico di Brera, via Bianchi 46, 23807 Merate, Italy \\ 6 Università degli Studi di Milano-Bicocca, Dipartimento di Fisica, Piazza delle Scienze 3, 20126 Milano, Italy \\ Department of Astronomy \& Astrophysics, Pennsylvania State University, University Park, PA 16802, USA \\ 8 NASA/Goddard Space Flight Center, Greenbelt, MD 20771, USA
}

Received 18 July 2006 / Accepted 2 October 2006

ABSTRACT

\begin{abstract}
Aims. We observed the high-energy peaked BL Lac object PKS 0548-322 (BZB J0550-3216) with Swift to study the temporal and spectral properties of its synchrotron emission simultaneously in the optical, ultraviolet, and X-ray energy bands.

Methods. We carried out a spectral analysis of 5 Swift XRT and UVOT observations of PKS 0548-322 taken over the period April-June 2005.

Results. The X-ray flux of this BL Lac source was found to be approximately constant at a level of $F_{(2-10 \mathrm{keV})} \simeq 4 \times 10^{-11} \mathrm{erg} \mathrm{cm}^{-2} \mathrm{~s}^{-1}$ which is a factor of 2 brighter than when observed by BeppoSAX in 1999 and close to the maximum intensity reported in the Einstein Slew Survey. The very good statistics obtained in the $0.3-10 \mathrm{keV}$ Swift X-ray spectrum allowed us to detect highly significant deviations from a simple power-law spectral distribution. A log-parabolic model describes the X-ray data well and gives a best-fit curvature parameter of 0.18 and a peak energy in the spectral energy distribution of about $2 \mathrm{keV}$. The UV spectral data from Swift UVOT join closely with a power-law extrapolation of the soft X-ray data points, suggesting that the same component is responsible for the observed emission in the two bands. The combination of synchrotron peak in the X-ray band and the high-intensity state confirms PKS $0548-322$ as a prime target for $\mathrm{TeV}$ observations. X-ray monitoring and coordinated $\mathrm{TeV}$ campaigns are highly advisable.
\end{abstract}

Key words. radiation mechanisms: non-thermal - galaxies: active - galaxies: BL Lacertae objects: individual: PKS 0548-322 X-rays: galaxies

\section{Introduction}

The BL Lacertae objects (BL Lacs) constitute a rather peculiar and extreme class of active galactic nuclei (AGNs). The main characteristics that distinguish BL Lacs are the rapid variability at all frequencies, high and variable radio and optical polarization, compact and flat-spectrum radio emission, superluminal motion, smooth and broad non-thermal continuum covering the electromagnetic spectrum from radio to $\gamma$-rays, and the almost complete absence of emission lines in the optical band. The extreme properties of BL Lacs are successfully explained in terms of relativistic beaming, i.e. of a relativistic bulk motion of the emitting region toward the observer (Blandford \& Rees 1978; Urry \& Padovani 1995).

The Spectral Energy Distribution (SED) of BL Lacs is generally characterized in a $\log (v F(v))-\log (v)$ representation by two emission peaks, the first produced by the synchrotron emission of relativistic electrons in a jet closely aligned to the line of sight, while inverse Compton scattering by the same population of relativistic electrons is thought to be at the origin of the higher energy peak (e.g. Ghisellini \& Maraschi 1989). BL Lacs are often divided into two classes according to the position of the synchrotron energy peak: low-energy peaked BL Lacs (LBLs), with the peak located at IR/optical wavelengths, and high-energy peaked BL Lacs (HBLs) with the synchrotron emission peaking in the UV/X-ray energy band (Giommi \& Padovani 1994; Padovani \& Giommi 1995).

Also named BZB J0550-3216 in the recent Multifrequency Catalogue of Blazars (Massaro et al. 2005), PKS 0548-322 ( $z=$ 0.069 , Fosbury \& Disney 1976) is a remarkable BL Lac object characterized by a relatively strong and fast variability in the X-ray energy band (Blustin et al. 2004). It is hosted in a giant elliptical galaxy (Falomo et al. 1995; Wurtz et al. 1996) that is the dominant member of a rich cluster of galaxies. The synchrotron power peaks in the X-ray band, and for this reason, it is classified as an HBL source (Padovani \& Giommi 1995). PKS 0548-322 was observed on several occasions by a number of X-ray astronomical satellites showing strong variations both in intensity and in spectral shape. A brief summary of the historical X-ray data on PKS 0548-322 can be found in Costamante et al. (2001) who also presented the analysis of three BeppoSAX observations. The brightest flux was reported in the Einstein Slew Survey (Perlman et al. 1996). The possibility that the X-ray spectrum of PKS 0548-322 might deviate from a simple power law has been apparent since the very early observations. Using Einstein data, 
Table 1. Swift observations and exposures of PKS 0548-322 in the 2nd quarter of 2005 .

\begin{tabular}{lccc}
\hline \hline Date & Start UT & $\begin{array}{c}\text { XRT Exp. } \\
(\mathrm{s})\end{array}$ & $\begin{array}{c}\text { UVOT Exp. } \\
(\mathrm{s})\end{array}$ \\
\hline April 1 & $00: 43$ & 1.258 & $/$ \\
April 26 & $23: 31$ & 5.212 & 7.791 \\
April 28 & $10: 53$ & 1.354 & $/$ \\
May 13 & $14: 22$ & 3.362 & 3.380 \\
May 21 & $10: 09$ & 9.224 & 9.135 \\
May 22 & $22: 52$ & 40.191 & $/$ \\
May 24 & $00: 02$ & 1.349 & 5.402 \\
May 26 & $00: 46$ & 400 & 1.926 \\
May 29 & $00: 07$ & 8.083 & 8.715 \\
June 14 & $16: 23$ & 1.235 & $/$ \\
June 24 & $17: 15$ & 7.951 & $/$ \\
\hline
\end{tabular}

Urry et al. (1986) interpreted the observed curvature as due to excess low-energy absorption, whereas Madejski (1985) suggested spectral bending. PKS 0548-322 was observed by EXOSAT at five epochs between 1983 and 1986 (Barr et al. 1988; Garilli \& Maccagni 1990), and broken power-law best fits gave a peak energy of the SED in the $2.5-5 \mathrm{keV}$ range. Similar results were obtained in the re-analysis of all EXOSAT observations by Ghosh \& Soundararajaperumal (1995), who noticed that a log-parabolic law represents the multifrequency spectrum of PKS 0548-322 well. A subsequent GINGA observation in February 1991 (Tashiro et al. 1995) showed a much flatter spectrum with an X-ray photon index of $1.84 \pm 0.02$ in the $2-30 \mathrm{keV}$ range, implying that the synchrotron peak in the SED had moved at energies higher than $30 \mathrm{keV}$. PKS 0548-322 was observed twice by BeppoSAX in 1999 on February 20 and on April 7. A first analysis was reported by Costamante et al. (2001), who found evidence of intrinsic curvature because a broken powerlaw model was necessary even in the presence of an extra absorption. The possibility of the presence of circumnuclear ionized gas was suggested by Sambruna \& Mushotzky (1998), who report evidence of an absorption feature around $0.6 \mathrm{keV}$ with a neutral hydrogen column density $N_{\mathrm{H}} \simeq 10^{21} \mathrm{~cm}^{-2}$; however, this feature was not confirmed by any subsequent spectroscopic XMM-Newton observation (Blustin et al. 2004). Finally, the Chandra detection of a diffuse soft X-ray radiation around PKS 0548-322, interpreted as a thermal emission of the host galaxy on a kpc scale, was reported by Donato et al. (2003).

This interesting source was observed on several occasions by the Swift satellite (Gehrels et al. 2004) from April to June 2005. In this paper we present the results of a detailed spectral analysis of the X-ray telescope (XRT, Burrows et al. 2005) and ultraviolet/optical telescope (UVOT, Roming et al. 2005) data, confirming that the X-ray spectrum of this HBL object shows a wellestablished curvature similar to the one found in other sources of the same type. In Sect. 2, the observations and the data reduction are presented, in Sect. 3 we describe the Swift XRT spectral analysis, and Sect. 4 is dedicated to the BeppoSAX data analysis. Finally the results are discussed in Sect. 5. Throughout this paper, errors are quoted at the $90 \%$ confidence level for the one parameter of interest $\left(\Delta \chi^{2}=2.7\right)$ unless otherwise specified.

\section{Observations}

As part of a Swift key project dedicated to the observation and monitoring of a sample of Blazars, PKS 0548-322 was pointed eleven times over the period April-June 2005. The journal of these observations is given in Table 1 , where we also report the net exposures with the XRT and UVOT instruments. On May 22, a deep (about $40 \mathrm{ks}$ ) XRT exposure of PKS 0548-322 was taken. The exposure times in the Burst Alert Telescope (BAT, Barthelmy et al. 2005) were not sufficient for detecting a source with a typical intensity lower than 2 mCrab like PKS 0548-322.

\subsection{XRT data reduction}

To obtain a signal with sufficient statistics for a detailed spectral analysis, we only considered observations longer than $5 \mathrm{ks}$. In particular, the X-ray spectrum accumulated during the longest XRT observation of May 22 had excellent photon statistics and allowed us to perform a very accurate spectral analysis. All XRT observations were carried out using the most sensitive photon counting readout mode (see Hill et al. 2004, for a description of readout modes). The XRT data were processed with the XRTDAS software package (v.1.8.0). Event files were calibrated and cleaned applying standard filtering criteria with the xrtpipeline task and using the latest calibration files available in the Swift CALDB distributed by HEASARC. Events in the energy range $0.3-10 \mathrm{keV}$ with grades $0-12$ were used in the analysis (see Burrows et al. 2005, for a definition of XRT event grades).

The source count rate was high enough to cause some photon pile-up in the inner 6 pixel $\left(\sim 14^{\prime \prime}\right)$ radius circle within the peak of the telescope point spread function (PSF), as derived from the comparison of the observed PSF profile with the analytical model derived by Moretti et al. (2005). We thus avoided pile-up effects selecting events within an annular region with an inner radius of 6 pixels and an outer radius of 30 pixels. The background was extracted from a nearby source-free circular region of 50 pixel radius. Ancillary response files for the spectral analysis were generated with the xrtmkarf task applying corrections for the PSF losses and CCD defects. The latest response matrices (v. 008) available in the Swift CALDB were used. The spectrum was binned to ensure a minimum of 20 counts per bin, and energy channels between $0.4 \mathrm{keV}$ and $0.6 \mathrm{keV}$ were excluded to avoid undesired effects on the measured spectral parameters due to residual instrumental features (Campana et al. 2006).

\subsection{UVOT data reduction}

UVOT observations with good exposure times were available for a number of pointings (see Table 1). Sky-corrected images were derived from the Swift archive, and aperture photometry was made with UVOTSOURCE using a $6^{\prime \prime}$ (12 pixels) radius for the $V, B, U$ filters and $12^{\prime \prime}$ for the $W 1, M 2$, and $W 2$ filters. Count rates in all the filters were always less than 5 counts/s, well below the pile-up threshold (10-15 counts/s).

The source showed no appreciable variations in all the pointings, with an average value $\langle B\rangle=16.9$. We report here only the data for the May 21 observation, which is the longest and is nearly simultaneous with the deep XRT observation of May 22.

The host galaxy of this source is a giant elliptical that has been extensively studied (e.g. by Falomo et al. 1995). To estimate the galaxy contribution within our $6^{\prime \prime}$ aperture, we integrated the best-fit de Vaucouleurs profile published by them (their Fig. 4) obtaining $R=15.7$. Assuming the typical colors for an elliptical galaxy $(B-V=0.96, V-R=0.61$; Fukugita et al. 1995), we derive $V=16.3$ and $B=17.2$. The AGN luminosity is therefore about $30 \%$ of the observed flux in these bands. For this reason we considered only photometric data in the $U$ and $U V$ bands where the host galaxy contribution is smaller. The 
Table 2. Best-fit spectral parameters of the log-parabolic model for the XRT (first section) and BeppoSAX (second section) observations of PKS 0548-322.

\begin{tabular}{|c|c|c|c|c|c|c|c|}
\hline Instrument & Date & $K^{(1)}$ & $a^{(1)}$ & $b^{(1)}$ & $\begin{array}{c}E_{\mathrm{p}} \\
(\mathrm{keV})\end{array}$ & $\begin{array}{c}F_{2-10}^{(2)} \\
\left(\mathrm{erg} \mathrm{cm}^{-2} \mathrm{~s}^{-1}\right) \\
\end{array}$ & $\chi_{\mathrm{r}}^{2} /$ d.o.f. \\
\hline Swift XRT & 2005 April 26 & $1.41(0.05) \times 10^{-2}$ & $1.81(0.07)$ & $0.18(0.13)$ & 3.4 & $4.0 \times 10^{-11}$ & $0.75 / 118$ \\
\hline \multirow[t]{2}{*}{ Swift XRT } & 2005 May 21 & $1.48(0.04) \times 10^{-2}$ & $1.71(0.05)$ & $0.38(0.10)$ & 2.4 & $4.0 \times 10^{-11}$ & $0.90 / 196$ \\
\hline & & $1.46(0.04) \times 10^{-2}$ & $1.78(0.03)$ & 0.18 frozen & 3.5 & $4.2 \times 10^{-11}$ & $0.94 / 197$ \\
\hline Swift XRT & 2005 May 22 & $1.66(0.02) \times 10^{-2}$ & $1.90(0.02)$ & $0.18(0.04)$ & 1.9 & $4.1 \times 10^{-11}$ & $1.03 / 408$ \\
\hline \multirow{2}{*}{ Swift XRT } & 2005 May 29 & $1.31(0.04) \times 10^{-2}$ & $1.77(0.06)$ & $0.34(0.11)$ & 2.2 & $3.3 \times 10^{-11}$ & $1.01 / 181$ \\
\hline & & $1.29(0.04) \times 10^{-2}$ & $1.83(0.04)$ & 0.18 frozen & 2.7 & $3.5 \times 10^{-11}$ & $1.03 / 182$ \\
\hline Swift XRT & 2005 June 24 & $1.52(0.05) \times 10^{-2}$ & $1.79(0.06)$ & $0.26(0.11)$ & 2.5 & $4.1 \times 10^{-11}$ & $1.09 / 183$ \\
\hline BeppoSAX LECS+MECS & 1999 Feb. 20 & $0.77(0.07) \times 10^{-2}$ & $1.57(0.10)$ & $0.48(0.10)$ & 2.8 & $2.3 \times 10^{-11}$ & $1.23 / 55$ \\
\hline BeppoSAX LECS+MECS & 1999 April 07 & $0.69(0.06) \times 10^{-2}$ & $1.82(0.10)$ & $0.42(0.10)$ & 1.6 & $1.5 \times 10^{-11}$ & $0.91 / 55$ \\
\hline
\end{tabular}

(1) Numbers in parenthesis are statistical errors at the $90 \%$ confidence level. ${ }^{(2)}$ Fluxes in the $2-10 \mathrm{keV}$ energy band are corrected for Galactic absorption.

measured magnitudes in the $U$ and $U V$ filters were $U=16.5$, $U V W 1=16.4, U V M 2=16.7$, and $U V W 2=16.6$. As the zeropoint uncertainty in the optical bands of UVOT is about $0.1 \mathrm{mag}$ (about 10\% in flux), an accurate estimate of the spectral shape is not currently feasible.

\section{The X-ray spectrum}

To study the X-ray spectral distributions of PKS 0548-322, we first considered the deep $40 \mathrm{ks}$ exposure performed on May 22. In Fig. 1 we show the $0.3-1.5 \mathrm{keV}$ (upper panel) and $1.5-10 \mathrm{keV}$ (middle panel) light curves of PKS 0548-322, together with the corresponding hardness ratio (lower panel). From the figure it is apparent that no significant temporal and spectral variability was present during the observation. We thus performed the spectral analysis using the events accumulated in the entire duration of the observation. We adopted the following two spectral models, a single power-law

$F(E)=K E^{-\alpha} \quad$ photons $\mathrm{cm}^{-2} \mathrm{~s}^{-1} \mathrm{keV}^{-1}$

and a log-parabolic law (Massaro et al. 2004a)

$F(E)=K E^{-(a+b \log E)} \quad$ photons $\mathrm{cm}^{-2} \mathrm{~s}^{-1} \mathrm{keV}^{-1}$

where the parameter $a$ is the photon index at $1 \mathrm{keV}$, while $b$ measures the curvature of the parabola. The latter model has the property of describing curved spectra with only one additional parameter with respect to the single power-law. The logparabolic model is also very useful for estimating the energy and the flux of the SED peak, simply given by, respectively:

$E_{\mathrm{p}}=10^{(2-a) / 2 b} \mathrm{keV}$,

$v_{\mathrm{p}} F\left(v_{\mathrm{p}}\right)=\left(1.60 \times 10^{-9}\right) K 10^{(2-a)^{2} / 4 b} \mathrm{erg} \mathrm{s}^{-1} \mathrm{~cm}^{-2}$.

The log-parabolic law generally fits the X-ray spectra of HBL sources well, as shown by Massaro et al. (2004a,b) for the cases of the X-ray spectra of Mkn 421 and Mkn 501. The relations between the parameters of synchrotron and inverseCompton radiation emitted by an electron population with a similar energy distribution have been recently investigated by Massaro et al. (2006).

When considering curved spectra, one parameter that needs special attention is the amount of low-energy neutral hydrogen absorbing column density $N_{\mathrm{H}}$. We first fitted the backgroundsubtracted $0.3-10 \mathrm{keV}$ spectrum with a single power-law model

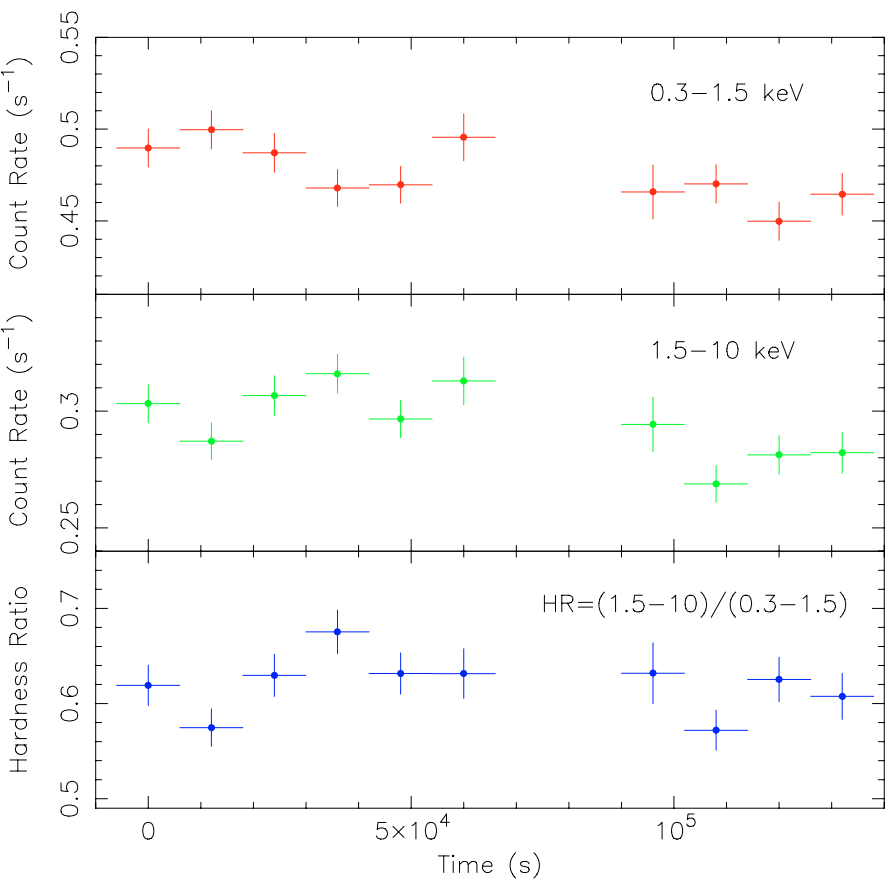

Fig. 1. Swift XRT 0.3-1.5 keV (upper panel) and 1.5-10 keV (middle panel) light curves of PKS 0548-322. In the lower panel the corresponding Hardness Ratio (HR) is plotted. Data are binned to $12 \mathrm{ks}$ resolution and error bars indicate statistical uncertainties at the $1 \sigma$ level.

with the absorption column density fixed at the known Galactic value of $N_{\mathrm{H}}=2.49 \times 10^{20} \mathrm{~cm}^{-2}$ (Murphy et al. 1996). We obtained a photon index of $1.96 \pm 0.02$ with a reduced $\chi_{\mathrm{r}}^{2}$ of 1.15 with 409 degree of freedom (d.o.f.). From the inspection of the residuals a clear systematic effect reflecting the presence of spectral curvature was observed. We thus adopted the log-parabolic model with the same amount of $N_{\mathrm{H}}$. The model provided a very good fit with $\chi_{\mathrm{r}}^{2}=1.03$ (408 d.o.f.), and an $\chi^{2}$ decrease of 50 for only one additional parameter. The $F$ test gives a probability of about $10^{-11}$ that this improvement is due to chance. Figure 2 shows the best-fit spectrum with this model and the residuals.

The same log-parabolic spectral law was then also applied to the analysis of the observations of shorter duration. The resulting best-fit parameters, the SED peak energies $E_{\mathrm{p}}$, and fluxes in the $2-10 \mathrm{keV}$ band are given in Table 2 . We note that for these shorter observations the parameters $a$ and $b$ are characterized by statistical uncertainties much larger than those of the 


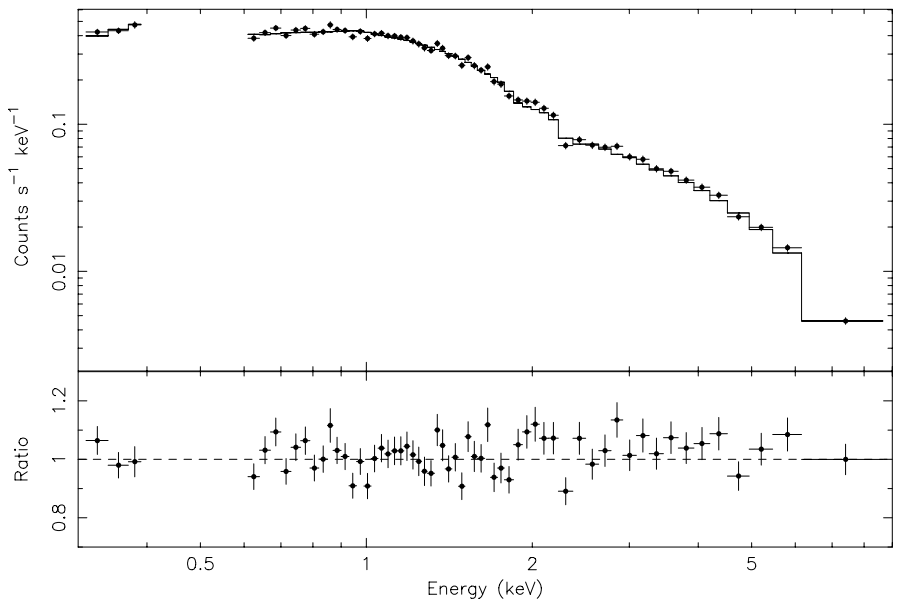

Fig. 2. Best-fit spectrum and residuals for the log-parabolic model of 0.3-10 keV XRT data (2005, May 22). Data points between 0.4 and $0.6 \mathrm{keV}$ were removed to avoid residual calibration uncertainties in this energy range.

May 22 observation. In two cases (April 26 and June 24), the value of $b$ was found to be consistent with the one measured on the May 22 deep observation, while it was larger on May 21 and 29 ( $b \sim 0.3-0.4)$. For these two pointings, best fits with the curvature $b$ frozen at 0.18 (the May 22 value) gave very small increases for the $\chi^{2}$ values (see Table 2), indicating that the higher curvature values measured are not statistically significant.

We also verified the possibility of an additional intrinsic absorption either in the nuclear environment of PKS 0548-322 or in the host elliptical galaxy. We recall that the exclusion of data points between 0.4 and $0.6 \mathrm{keV}$ do not allow an accurate estimation of the absorption. A simple power-law best fit with a free $N_{\mathrm{H}}$ for the May 22 observation resulted in a slightly higher absorption column density of $(4.2 \pm 0.6) \times 10^{20} \mathrm{~cm}^{-2}$. However, the $\chi^{2}$ was found to be comparable to the fit with $N_{\mathrm{H}}$ fixed at the Galactic value $\left(\chi_{\mathrm{r}}^{2} /\right.$ d.o.f. $\left.=1.14 / 408\right)$ and, again, the residuals showed a clear spectral curvature. The May 22 spectrum was also fit with the log-parabolic model leaving $N_{\mathrm{H}}$ free to vary. The fit was good $\left(\chi_{\mathrm{r}}^{2} /\right.$ d.o.f. $\left.=1.03 / 407\right)$ and statistically equivalent to the case with fixed Galactic absorption. We obtained an absorption column density of $(2.1 \pm 1.0) \times$ $10^{20} \mathrm{~cm}^{-2}$, in agreement with the Galactic value, and a curvature $b=0.21 \pm 0.10$. Therefore, we conclude that the X-ray spectrum of PKS 0548-322 is intrinsically curved and that it is described by a log-parabolic model well with no excess absorption required, like the two well-known HBL sources Mkn 421 and Mkn 501.

\section{BeppoSAX observations}

In this section we present a re-analysis of BeppoSAX data considering the log-parabolic model, not used by Costamante et al. (2001), to evaluate the spectral curvature and compare it to that seen by the XRT in different brightness states.

In the two BeppoSAX observations in 1999 (February 20 and on April 7), PKS 0548-322 was too faint to be detected by the PDS instrument, so we analyzed only LECS and MECS data. Events for spectral analysis were selected in circular regions of $6^{\prime}$ and $4^{\prime}$ and in the energy bands $0.1-2.0 \mathrm{keV}$ and 2.0-10.0 keV for the LECS and MECS, respectively. Background spectra were taken from the blank field archive at the ASI Science Data Center. In Table 2 we also report the logparabolic best-fit parameters of these BeppoSAX observations.

We first note that in 1999 the typical $2-10 \mathrm{keV}$ flux of PKS 0548-322 was about a factor of 2 lower than in 2005. This change in luminosity was accompanied by a different spectral curvature ( $b \simeq 0.45$ ), while the peak energy appears stabler. To verify that this change of curvature is real, we fit the BeppoSAX data with $b$ kept frozen to the value measured by Swift in the May 22 observation. We found an increase in $\chi^{2}$ corresponding to an $F$-test probability of $4 \times 10^{-6}$ and $2 \times$ $10^{-5}$ for the observations of February 20 and April 7, respectively. These results make us confident that the change in curvature is very significant. We also verified the possibility of having an extra-absorption in the local frame of PKS 0548-322. The two BeppoSAX 1999 spectra were fit with the log-parabolic model with free $N_{\mathrm{H}}$. In both pointings we found an absorption column density of $N_{\mathrm{H}}=(3.2 \pm 1.2) \times 10^{20} \mathrm{~cm}^{-2}$, consistent with the Galactic value, and curvatures $b=0.39 \pm 0.18$ and $b=0.32 \pm 0.20$ for the February 20 and April 7 observations, respectively. Moreover, the fits were statistically equivalent to the ones with fixed absorption. We conclude that the X-ray spectrum of PKS 0548-322 was also intrinsically curved in the two BeppoSAX 1999 observations and is well-described by a log-parabolic model with Galactic absorption.

\section{Discussion}

Our spectral analysis of the recent series of Swift XRT observations of PKS 0548-322 has shown that the X-ray spectrum of this BL Lac object is characterized by a significant curvature that is well-fitted by a log-parabolic law with a curvature parameter and peak energy similar to those of other HBL sources (e.g. Massaro et al. 2004a; Giommi et al. 2002; Tramacere et al. 2006). Blustin et al. (2004) summarize some historical X-ray data for PKS 0548-322 since its first X-ray observation on March 1979 and find that the $2-10 \mathrm{keV}$ flux in this source was always within the range $(\sim 1.5-\sim 4.5) \times 10^{-11} \mathrm{erg} \mathrm{cm}^{-2} \mathrm{~s}^{-1}$. The Swift XRT observations in the $2^{\text {nd }}$ quarter of 2005 described in this paper confirm this rather stable behavior with observed fluxes close to the historical maximum. This relative stability at X-ray frequencies of PKS 0548-322 contrasts with the behavior of the Mkn 421 and Mkn 501 (Fossati et al. 2000; Massaro et al. 2004a,b) whose X-ray fluxes varied well over an order of magnitude. Of course, the sampling of this historic X-ray data set is rather poor, and we cannot exclude the occurrence of large outbursts on a time scale of a few months, possibly associated with spectral changes.

Figure 3 shows the SEDs of PKS 0548-322 in the UV to X-ray range for the long May 22 pointing and the two BeppoSAX observations. The main differences between the recent high state with respect to the previous data is the very apparent decrease of the curvature, whereas the peak energy is around the same values. Moreover, the UV data can be connected to the low-energy $\mathrm{X}$-ray points by a powerlaw interpolation, suggesting that they come from the same component. Simultaneous UV data are not available for the BeppoSAX observations; however, a low-energy extrapolation of the X-ray SEDs indicates that at those epochs the UV emission of PKS 0548-322 should have been lower than found in 2005. A recent extensive study of a large set of X-ray observations of Mkn 421 (Massaro et al. 2004a; Tramacere et al. 2006b) has shown that this HBL source shows a positive correlation 


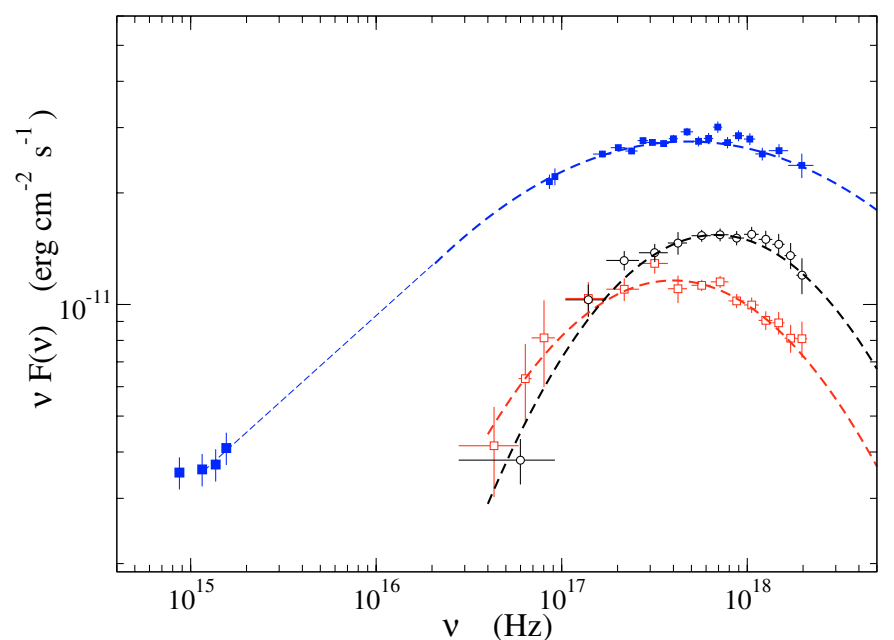

Fig. 3. Optical, UV, and X-ray SED of PKS 0548-322 from BeppoSAX (1999 February 20: open circles; 1999 April 07: open squares), Swift XRT (2005 May 22, filled circles), and Swift UVOT (2005 May 21, filled squares) observations. The thick dashed lines represent the logparabolic best-fit models to the X-ray data points. The thin dashed line is a power-law extrapolation of the UV spectral data to the soft X-ray band. Error bars indicate statistical uncertainties at the $1 \sigma$ level. The host galaxy contribution has not been subtracted from UVOT data (see Sect. 2.2).

between the energy and the flux at the SED peak. In the case of PKS 0548-322, we do not observe a significant increase in the peak energy with flux. This finding can be an indication that statistical acceleration works with different efficiencies in these two sources.

PKS 0548-322 is a good candidate for a detection in the TeV range, as already noticed by Costamante \& Ghisellini (2002). In a synchrotron self-Compton scenario, a brightening at X-ray frequencies often corresponds to an enhanced TeV luminosity. It is therefore very useful to organize a monitoring program of the $\mathrm{X}$-ray flux in the next months to trigger a possible TeV detection. The study of the evolution of both synchrotron and inverse Compton components in the SED will be very useful for understanding the physical conditions in the nuclear region and, when compared with other TeV BL Lacs, for deriving a more general picture of this class of sources. Thanks to the wide field of view of the BAT instrument and to its fast pointing capability with the UVOT and XRT narrow-field telescopes, Swift is the satellite best-suited to perform such a program.
Acknowledgements. We are grateful to the referee for his/her useful comments and suggestions. The authors acknowledge the financial support of ASDC by the Italian Space Agency (ASI) and of the Phys. Dept. by Università di Roma La Sapienza.

\section{References}

Barr, P., Giommi, P., \& Maccagni, D. 1988, ApJ, 324, L11 Barthelmy, S. D., et al. 2005, Space Science Rev., 120, in press [arXiv:astro-ph/0507410]

Blandford, R. D., \& Rees, M. J. 1978, in Pittsburgh Conference on BL Lac Objects, ed. A. N. Wolfe (Pittsburgh: University of Pittsburgh Press), 328

Blustin, A., Page, M., \& Branduardi-Raymont, G. 2004, A\&A, 417, 61

Burrows, D. N., Hill, J. E., Nousek, J. A., et al. 2005, Space Sci. Rev., 120, 165

Campana, S., Beardmore, A. P., Cusumano, G., \& Godet, O. 2006, Swift-XRTCALDB-09, http://swift.gsfc.nasa.gov/docs/heasarc/caldb/ swift/docs/xrt/SWIFT-XRT-CALDB-09.pdf

Cardelli, J. A., Clayton, G. C., \& Mathis, J. S. 1989, ApJ, 345, 245

Costamante, L., \& Ghisellini, G. 2002, A\&A, 384, 56

Costamante, L., Ghisellini, G., Giommi, P., et al. 2001, A\&A, 371, 512

Donato, D., Gliozzi, M., Sambruna, R. M., \& Pesce, J. E. 2003, A\&A, 407, 503

Falomo, R., Pesce, J. E., \& Treves, A. 1995, ApJ, 438, L9

Fosbury, R., \& Disney, M. 1976, ApJ, 207, L75

Fossati, G., Celotti, A., Chiaberge, M., et al. 2000, ApJ, 541, 166

Fukugita, M., Shimazaku, K., \& Ichikawa, T. 1995, PASP, 107, 945

Garilli, B., \& Maccagni, D. 1990, A\&A, 229, 88

Gehrels, N., Chincarini, G., Giommi, P., et al. 2004, ApJ, 611, 1005

Ghisellini, G., \& Maraschi, L. 1989, ApJ, 340, 181

Ghosh, K., \& Soundararajaperumal, S. 1995, ApJS, 100, 37

Giommi, P., \& Padovani, P. 1994, MNRAS, 268, L51

Giommi, P., Capalbi, M., Fiocchi, M., et al. 2002, in Blazar Astrophysics with BeppoSAX and Other Observatories, ed. P. Giommi, E. Massaro, \& G. Palumbo, 63

Hill, J. E., Burrows, D. N., Nousek, J. A., et al. 2004, Proc. SPIE, 5165, 217

Li, W., Jha, S., Filippenko, A. V., et al. 2005, PASP, in press

Madejski, G. 1985, Ph.D. Thesis, Harvard University

Massaro, E., Perri, M., Giommi, P., \& Nesci, R. 2004a, A\&A, 413, 489

Massaro, E., Perri, M., Giommi, P., Nesci, R., \& Verrecchia, F. 2004b, A\&A, 422, 103

Massaro, E., Sclavi, S., Giommi, P., Perri, M., \& Piranomonte, S. 2005, Multifrequency Catalogue of Blazars, Vol. I (Roma: Aracne editrice)

Massaro, E., Tramacere, A., Perri, M., et al. 2006, A\&A, 448, 861

Moretti, A., Campana, S., Mineo, T., et al. 2005, Proc. SPIE, 5898, 360

Murphy, E., Lockman, F., Laor, A., \& Elvis, M. 1996, ApJS, 105, 369

Padovani, P., \& Giommi, P. 1995, ApJ, 444, 567

Perlman, E. S., Stocke, J. T., Schachter, J. F., et al. 1996, ApJS, 104, 251

Roming, P. W. A., Kennedy, T. E., Mason, K. O., et al. 2005, Space Sci. Rev., 120,95

Sambruna, R., \& Mushotzky, R. 1998, ApJ, 502, 630

Smith, P. S., Jannuzi, B. T., \& Richard, E. 1991, ApJS, 77, 67

Tashiro, M., Makishima, K., Ohashi, T., et al. 1995, PASJ, 47, 131

Tramacere, A., et al. 2006, in preparation

Tramacere, A., Massaro, F., \& Cavaliere, A. 2006, in preparation

Urry, C. M., \& Padovani, P. 1995, PASP, 107, 803

Urry, C., Mushotzky, R., \& Holt, S. 1986, ApJ, 305, 369

Wurtz, R., Stocke, J. T., \& Yee, H. K. C. 1996, ApJS, 103, 109 